\title{
Ectromelia virus induces tubulin cytoskeletal rearrangement in immune cells accompanied by a loss of the microtubule organizing center and increased a-tubulin acetylation
}

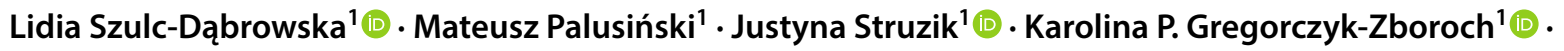 \\ Felix N. Toka ${ }^{1,2}$ - Ada Schollenberger ${ }^{1} \cdot$ Małgorzata Gieryńska $^{1}$ (i)
}

Received: 26 May 2018 / Accepted: 9 September 2018 / Published online: 29 October 2018

(C) The Author(s) 2018

\begin{abstract}
Ectromelia virus (ECTV) is an orthopoxvirus that productively replicates in dendritic cells (DCs), but its influence on the microtubule (MT) cytoskeleton in DCs is not known. Here, we show that ECTV infection of primary murine granulocytemacrophage colony stimulating factor-derived bone marrow cells (GM-BM) downregulates numerous genes engaged in MT cytoskeleton organization and dynamics. In infected cells, the MT cytoskeleton undergoes dramatic rearrangement and relaxation, accompanied by disappearance of the microtubule organizing centre (MTOC) and increased acetylation and stabilization of MTs, which are exploited by progeny virions for intracellular transport. This indicates a strong ability of ECTV to subvert the MT cytoskeleton of highly specialized immune cells.
\end{abstract}

Ectromelia virus (ECTV) belongs to the genus Orthopoxvirus of the family Poxviridae, which also includes variola virus (VARV), vaccinia virus (VACV), cowpox virus (CPXV) and monkeypox virus (MPXV). ECTV is a natural pathogen of mice that causes mousepox, a severe disease with high mortality rates in certain strains of mice. Mousepox is considered a model for smallpox and other acute generalized viral infections, and for evaluation of anti-poxvirus drugs and vaccination efficacy [1,2]. Currently, orthopoxviruses pose a threat to livestock animals and wildlife and are the source of zoonoses in humans and companion animals (VACV, CPXV and MPXV) [3-5].

Microtubules (MTs) form a dynamic network of filaments that are important in a wide range of cellular processes, including intracellular transport of organelles, mitosis, cell

Handling Editor: William G Dundon.

Lidia Szulc-Dąbrowska

lidia_szulc@sggw.pl

1 Division of Immunology, Department of Preclinical Sciences, Faculty of Veterinary Medicine, Warsaw University of Life Sciences-SGGW, Ciszewskiego 8, 02-786 Warsaw, Poland

2 Center for Integrative Mammalian Research, Ross University School of Veterinary Medicine, PO Box 334, Basseterre, St. Kitts and Nevis migration and polarization. They nucleate from the MT organizing center (MTOC), which includes centrosomes. MTs are characterized by dynamic instability, a process involving repeated phases of growth (polymerization) and shrinkage (disassembly), separated by pauses, catastrophes and rescues [6]. In response to environmental signals, subsets of MTs can become stabilized and long-lived through post-translational modifications, such as detyrosination and acetylation. MT dynamics and organization can be modulated by different signaling cues, such as viruses, which exploit MT not only for intracellular trafficking and formation of replication centers but also for controlling the behavior of the infected cell for their own benefit [7].

In non-immune cells, orthopoxviruses exploit both the actin and MT cytoskeleton for efficient penetration, replication, transport and release of progeny viral particles [8-10]. However, orthopoxviruses, such as VACV and CPXV, are not able to replicate productively in immune dendritic cells (DCs) [11-13], and therefore it is not known if orthopoxviruses have an influence on the cytoskeleton of DCs, which represent the most potent cells in stimulation of an antigenspecific T-cell response.

Our experiments were conducted on granulocyte-macrophage colony-stimulating factor (GM-CSF)-cultured bone marrow cells (GM-BM) composed of conventional DCs (cDCs) and macrophages. GM-BM cells were generated from bone marrow precursors obtained from femurs and 
tibias of BALB/c mice with ethical approval as described previously $[14,15]$. After 8 days of culture, cDCs were enriched by magnetic-activated cell sorting (MACS) using CD11 $\mathrm{c}^{+}$-labeled magnetic beads (Miltenyi Biotec) and the surface expression of CD11c, CD11b, MHC II and CD205 molecules was assessed by flow cytometry as reported previously $[14,15]$. CD11c-enriched GM-BM cells were left uninfected (mock) or were infected with ECTV (ATCC VR-1374) at a multiplicity of infection (MOI) of 1 and/or were treated with $1 \mu \mathrm{g}$ of lipopolysaccharide (LPS; Escherichia coli 0111:B4; Sigma-Aldrich) per ml for $24 \mathrm{~h}$.

Expression of tubulin- and actin-cytoskeleton-related genes was evaluated using quantitative real-time PCR (qPCR) analysis. RNA isolation and reverse transcription (RT) were performed as described previously $[14,15]$. Realtime PCR was performed using Real-Time Ready Custom Panel 96 pre-coated plates and LightCycler 480 Probes Master Mix (Roche Diagnostics) according to the manufacturer's instructions. Using a LightCycler 96 instrument (Roche Diagnostics), qPCR was performed as follows: a preincubation step at $95^{\circ} \mathrm{C}$ for $10 \mathrm{~min}$, then 45 cycles at $95^{\circ} \mathrm{C}$ for $10 \mathrm{~s}, 60^{\circ} \mathrm{C}$ for $30 \mathrm{~s}$ and $72^{\circ} \mathrm{C}$ for $1 \mathrm{~s}$, followed by a final cooling step at $40^{\circ} \mathrm{C}$ for $30 \mathrm{~s}$. The gene expression levels were quantified by the $\Delta \Delta \mathrm{Ct}$ method using $C d k n l a$ as an endogenous control.

Immunofluorescence staining was performed in GM-BM cells as described previously [9]. Statistical analysis was performed using Statistica 6.0 software (Statsoft) using the Mann-Whitney U-test or Student's $t$-test (*, $p<0.05$; **, $p<0.01$, and $* * *, p<0.001$ ).

After MACS separation, GM-BM cells were enriched for cells expressing high levels of CD11c (Fig. 1A) and MHC II (Fig. 1B) molecules, and thereby resembling a cDC phenotype. Within the cytoplasm of MHC II ${ }^{\text {high }}$ GM-BM cells, replication centers called "viral factories" were formed upon ECTV infection (Fig. 1B). These highly organized compartments were simultaneously stained with Hoechst 33342 for detection of viral DNA and anti-ECTV pAbs for detection of viral antigens (Fig. 1C). Our previous studies have shown that ECTV is able to replicate productively in GM-BM cells, including cDCs derived from BALB/c or C57BL/6 mice [14, 15]. At $4 \mathrm{hpi}$, the cells developed several "viral factories", which increased in size during later stages of infection (24 hpi). Moreover, at $24 \mathrm{hpi}$, the cytoplasm of GM-BM cells was filled with numerous progeny virions (Fig. 1C), which were ultimately released from the infected cells [14]. The productive replication cycle of ECTV in GM-BM cells was further confirmed using a plaque assay, which detected an approximately 500-fold increase in the number of infectious viral particles between 4 and 24 hpi [15].

Real-time PCR analysis of tubulin- and actin-cytoskeleton-related gene expression revealed that GM-BM cells infected with ECTV at $24 \mathrm{hpi}$ had a profound downregulation of several genes encoding proteins involved in cytoskeleton organization and dynamics (Fig. 1D). Among the tubulincytoskeleton-related genes, ECTV significantly $(p<0.001)$ decreased the expression of Clasp2 (CLIP-associated protein 2), Clip1 (CAP-GLY domain-containing linker protein 1), Mapre1 (MT-associated protein, RP/EB family, member 1), Racgapl (Rac GTPase-activating protein 1) and Stmnl (stathmin 1). CLASP2 is a MT plus-end tracking protein (+TIP) that promotes directionally persistent migration and stabilizes MTs, specifically at the leading edge of migrating cells [16]. CLIP1 is another + TIP protein that promotes MT growth and bundling and plays an important role in intracellular vesicle transport through linking of cytoplasmic vesicles to MTs [17]. MAPRE1, also known as endbinding protein 1 (EB1), associates with the plus-ends of growing MTs and acts as an adaptor to recruit and bind other +TIP proteins [18]. Meanwhile, RacGAP1 (also known as MgcRacGAP or hCYK-4) is a component of the central spindle and midbody, and plays a key role in controlling cytokinesis. Additionally, RacGAP1 can inactivate Rho proteins, such as Rac and Cdc42, and it therefore modulates cell morphology, motility and polarization [19]. Stathmin plays a critical role in regulation of MT dynamics during cell-cycle progression by promoting MT depolymerization and/or preventing polymerization of tubulin heterodimers [20]. Inhibition of stathmin expression causes an increase in the content of polymerized MTs, interferes with mitotic progression, and contributes to severe mitotic spindle abnormalities [21]. Taken together, our data suggest that ECTV infection of GM-BM cells might lead to modulation of MT dynamics and therefore might alter MT-dependent processes such as cell division, organelle transport, cell polarity, and cytoskeletal remodeling for cell migration.

Additionally, among the actin-cytoskeleton-related genes ECTV significantly $(p<0.001)$ reduced the expression of Actr2 (ARP2 actin-related protein 2), Arpc3 (actinrelated protein $2 / 3$ complex, subunit 3), Cfll (cofilin 1, non-muscle), Ezr (ezrin) and Vasp (vasodilator-stimulated phosphoprotein) in GM-BM cells at 24 hpi (Fig. 1D). ACTR2 and ARPC3 are two proteins of the seven-subunit protein complex ARP2/3, which is involved in regulation of actin filament nucleation and polymerization during important cellular processes, such as lamellipodia extension formation, vesicular trafficking, and phagocytosis [22]. It has been shown that depletion of the ARP $2 / 3$ complex in mouse embryonic fibroblasts inhibits lamellipodia formation and influences the directionality of cell movement. In the absence of the ARP2/3 complex, however, cells are able to form excessive filopodial protrusions that are responsible for defective random cell motility [23]. Because $C f l 1, E z r$ and Vasp also regulate actin assembly/disassembly and/ or cell motility [24-26], we suggest that actin cytoskeletal dynamics might also be dramatically altered during ECTV 
A

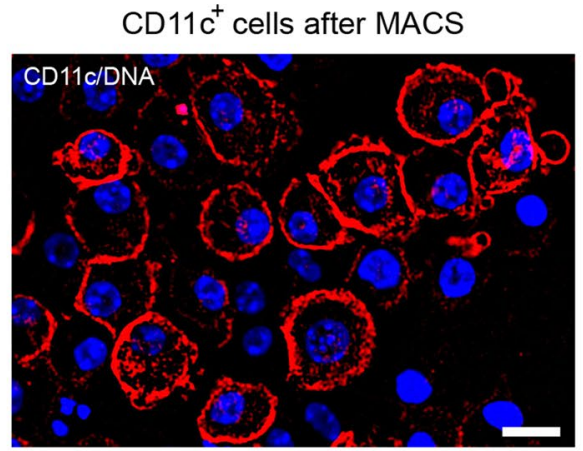

B

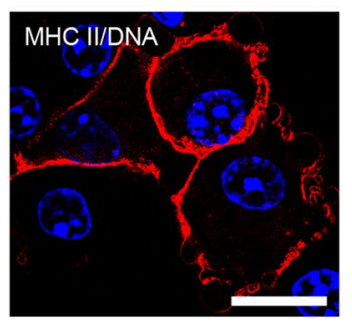

\section{ECTV}

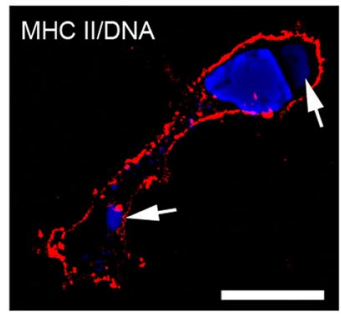

C
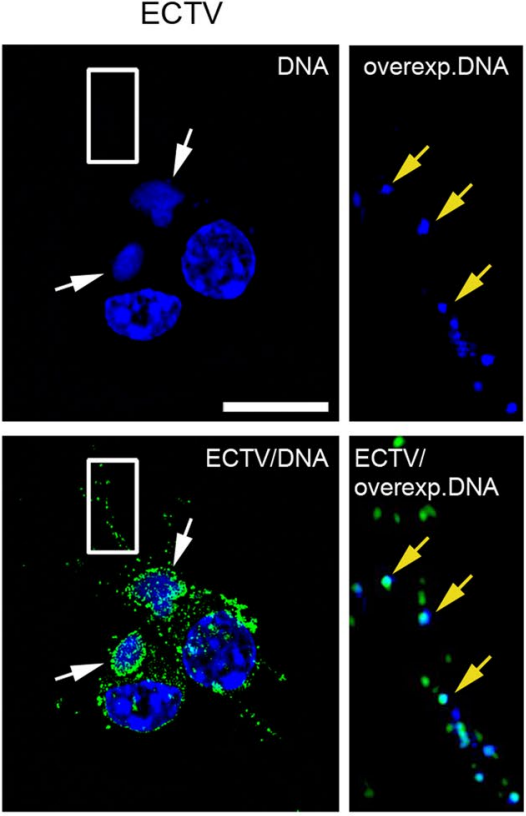

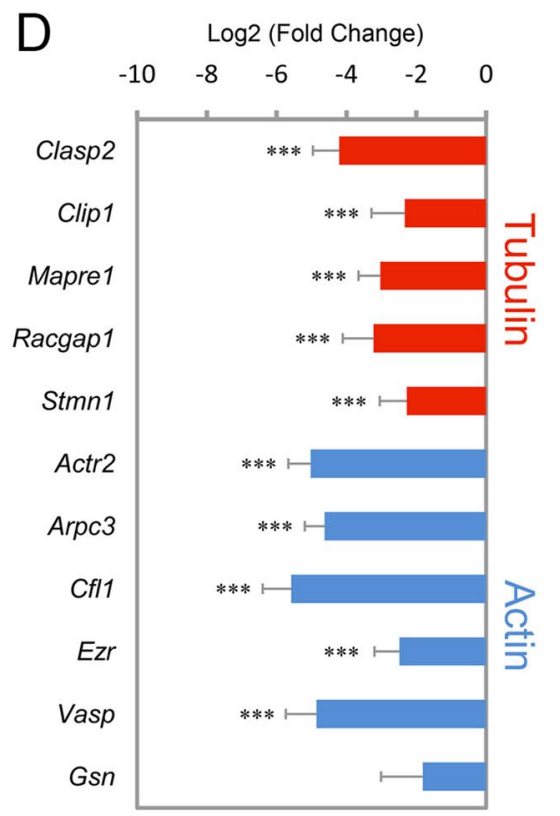

E

Mock
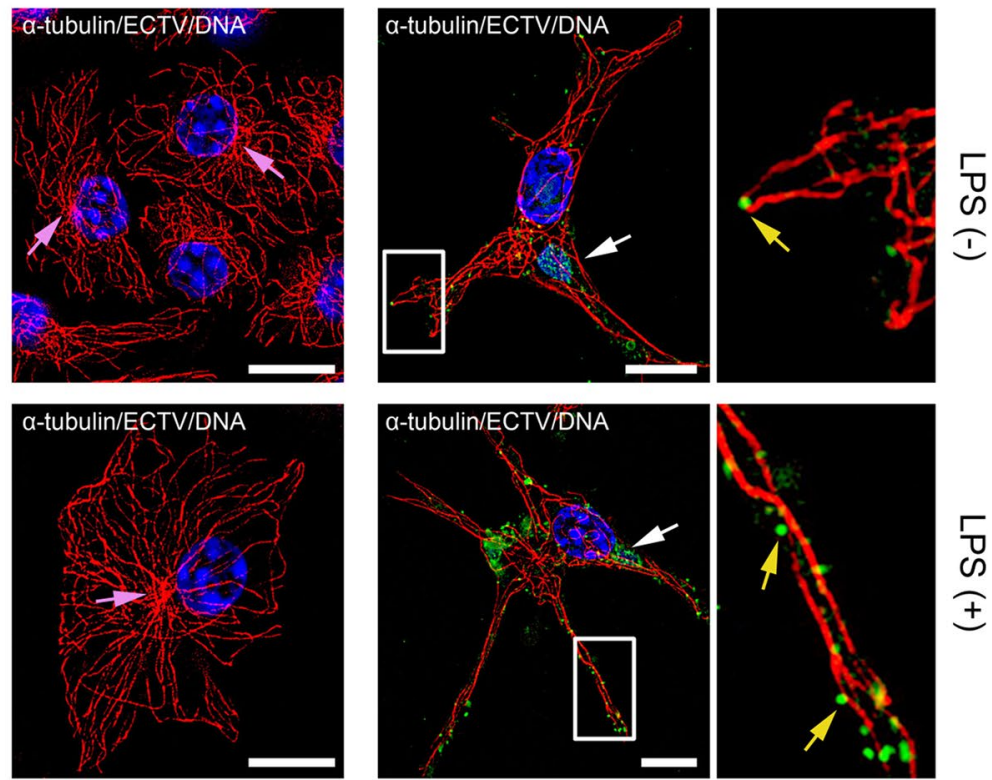

Fig. 1 ECTV infection downregulates tubulin-related genes and remodels the microtubule cytoskeleton in GM-BM cells. (A) Enrichment of CD11 $\mathrm{c}^{\text {high }}$ cells after MACS separation. The cells were stained for CD11c (red fluorescence) and nuclear DNA (blue fluorescence). Scale bar $=10 \mu \mathrm{m}$. (B) The expression of MHC II molecules on enriched mock- and ECTV-infected CD11 $\mathrm{c}^{\text {high }}$ GM-BM cells. MHC II molecules (red fluorescence), nuclear DNA (blue fluorescence). Scale bars $=10 \mu \mathrm{m}$. White arrows indicate viral factories. (C) Representative images of ECTV-infected GM-BM cells stained with Hoechst 33342 (blue fluorescence) and pAbs anti-ECTV (green fluorescence). The magnified images are of the boxed regions. Arrows indicate viral factories (white) and viral particles (yellow). Overexp. DNA, overexposed DNA. Scale bar $=10 \mu \mathrm{m}$. (D) Log2 fold change of mRNA expression for tubulin- and actin-related genes in GM-BM cells following infection with ECTV at $24 \mathrm{hpi}$ from three independent experiments (Student's $t$-test; $* * *, p \leq 0.001$ ). (E) Morphology of microtubule cytoskeleton in mock- and ECTV-infected GM-BM cells at $24 \mathrm{hpi}$. The cells were stained for $\alpha$-tubulin (red fluorescence), viral antigen (green fluorescence), and nuclear and viral DNA (blue fluorescence). Arrows indicate MTOC (purple), viral factories (white) and viral particles (yellow). The magnified images are of the boxed regions. Scale bars $=10 \mu \mathrm{m}$ 

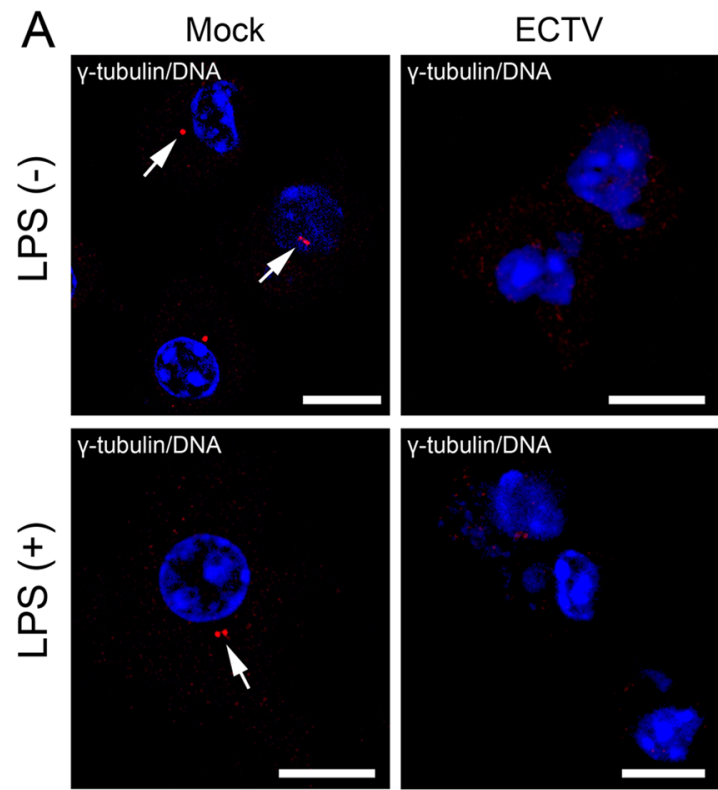

Fig. 2 ECTV alters the MTOC organization in infected GM-BM cells at 24 hpi. (A) Representative images of mock- and ECTV-infected GM-BM cells that were left untreated or treated with LPS for 24 h. $\gamma$-tubulin, red; viral antigen, green; nuclear and viral DNA, blue.

infection in GM-BM cells. Despite downregulation of critical genes regulating actin cytoskeleton organization and dynamics, ECTV-infected GM-BM cells are able to form long actin-based cellular extensions [27]. Long actin-based filopodial extensions are also observed in ECTV-infected L929 fibroblasts, which exhibit impaired cell migration [9].

Next, we performed morphological studies to examine the architecture of the MT cytoskeleton in infected GM-BM cells using immunofluorescence staining and microscopy analysis. GM-BM cells, either untreated (immature) or treated with LPS (mature), showed the presence of intertwined MT filaments, which radiated from the MTOC located at the perinuclear region (Fig. 1E). After LPS challenge, however, the MT network was more "relaxed" compared to LPS-untreated GM-BM cells. The relaxation of MT filaments was intensified in both ECTV-infected LPS-treated and untreated cells (Fig. 1E). In these cells, MTs were loosely arranged and were less intertwined and straighter, especially within long cellular extensions. The changes in the MT cytoskeleton organization were accompanied by colocalization of progeny viral particles with MT filaments, especially at the cell periphery. This suggests that newly synthesized ECTV particles utilize MTs for their intracellular transport to the plasma membrane, similar to VACV in rabbit kidney $\mathrm{RK}_{13}$ cells [28]. VACV encodes MT-associated proteins (MAPs) such as A10 and L4 that are responsible for direct binding of viral cores to MTs in vitro [29]. Moreover, A36 protein, which is found in the membrane of intracellular enveloped virus (IEV) of VACV and ECTV, mediates
B

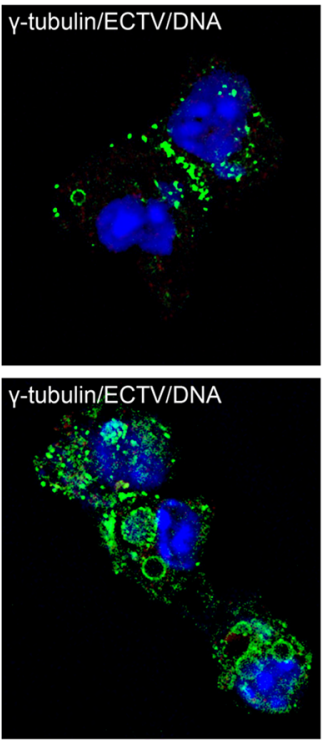

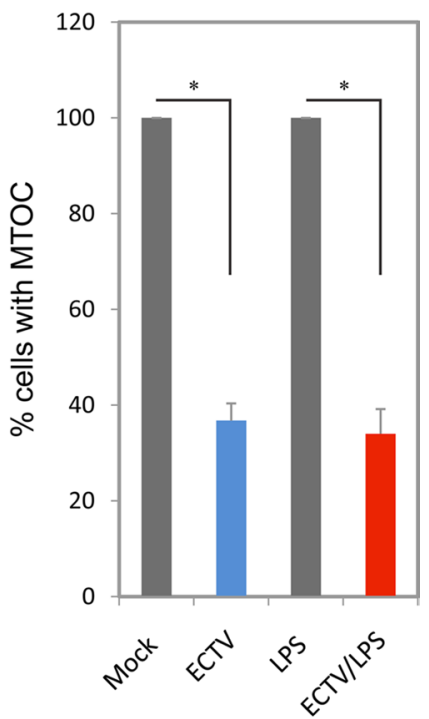

White arrows indicate MTOC. Scale bars $=10 \mu \mathrm{m}$. (B) The mean percentage of cells $( \pm$ SD) with MTOC analyzed in 100 cells per experimental condition from three independent experiments (Mann-Whitney $\mathrm{U}$ test; $*, p \leq 0.05$ )

MT-dependent intracellular movement of IEV and actin tail formation [30]. It has been reported that ECTV devoid of A36 exhibits reduced MT-mediated transport [31].

The rearrangement of the MT cytoskeleton architecture in ECTV-infected GM-BM cells was also manifested by disruption of the MTOC. In infected cells, MT filaments lost centrosomal focus at the perinuclear region, suggesting MTOC disorganization (Fig. 1E). The loss of MTOC structure was further confirmed by immunofluorescence staining of centrosomes with anti- $\gamma$-tubulin antibodies (Fig. 2A). In uninfected immature and mature GM-BM cells, the centrosome was clearly visible in the perinuclear region. At the same time, ECTV infection resulted in a dramatic reduction of $\gamma$-tubulin labeling, and only $36 \%$ and $34 \%$ of all infected untreated and LPS-treated GM-BM cells, respectively, showed the presence of $\gamma$-tubulin in centrosomes (Fig. 2B). Altered centrosome organization was also observed in L929 fibroblasts [9] and RAW 264.7 macrophages [32] during the later stages of ECTV infection, suggesting that it can be a generalized cell-independent phenomenon occurring during productive viral replication. In addition, VACV infection of HeLa cells resulted in severe reduction of $\gamma$-tubulin, as well as other centrosome/centriole components, such as pericentrin, C-Nap 1, Nek 2, and centrin, leading to the loss of centrosomal microtubule nucleation efficiency. It is assumed that the loss of centrosome function enhances disruption of the MT cytoskeleton during infection [29].

Deregulation of centrosome function may serve multiple roles important for virus morphogenesis, spread and survival 

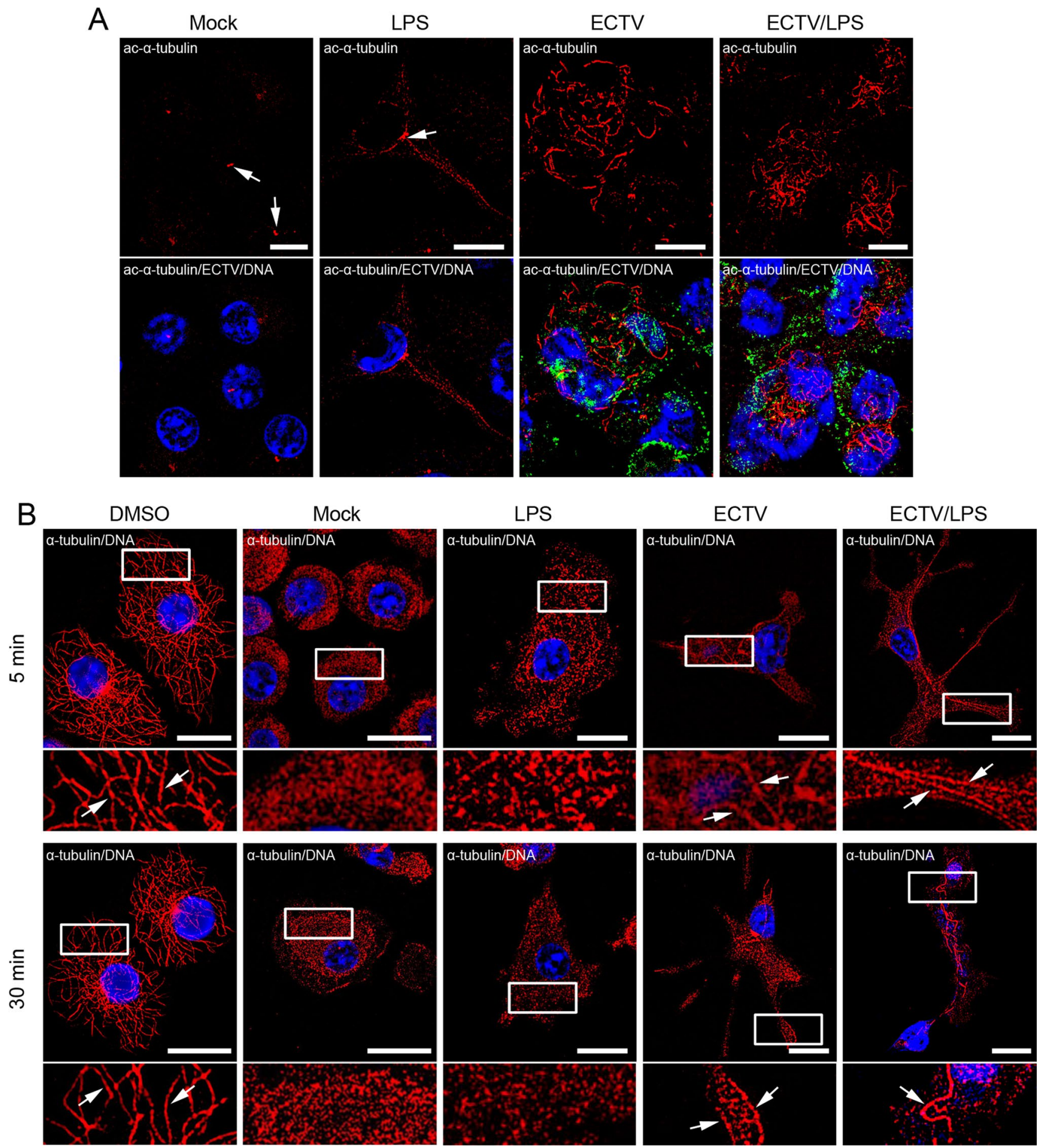

Fig. 3 GM-BM cells infected with ECTV display increased acetylation of $\alpha$-tubulin and higher resistance to nocodazole treatment. (A) Representative images showing acetylated $\alpha$-tubulin in mock- and ECTV-infected GM-BM cells that were left untreated or treated with LPS at 24 hpi. Acetylated $\alpha$-tubulin, red; viral antigen, green; nuclear and viral DNA, blue. White arrows indicate MTOC; Scale bars $=10 \mu \mathrm{m}$. (B) GM-BM cells, incubated in the presence of nocodazole for 5 and $30 \mathrm{~min}$, were stained for $\alpha$-tubulin (red), and nuclear and viral DNA (blue). White arrows indicate microtubules. DMSO, dimethyl sulfoxide. Scale bars $=10 \mu \mathrm{m}$ 
[7]. It has been shown that herpes simplex virus type 1 (HSV1) disrupts the centrosome, and thus switches MT nucleation to the trans-Golgi network (TGN) for efficient assembly and subsequent egress of progeny virions. Moreover, targeting the centrosome function may inhibit the innate antiviral response, because viral components may be recognized by the cell as foreign or misfolded proteins and then are transported along MTs to the aggresomes/proteolytic centers (located at the centrosome) for degradation by proteasomes and/or autophagy. Because the MTOC serves as a source of substrates for proteasomes and participates in antigen processing, viruses may disrupt centrosome function to modulate the adaptive antiviral response [33].

Lastly, we addressed the impact of ECTV infection on acetylation of $\alpha$-tubulin in GM-BM cells. In uninfected cells, acetylated $\alpha$-tubulin was observed almost exclusively in centrioles, whereas LPS treatment increased acetylation of MTs (Fig. 3A). This observation is in line with a previous study showing extensive acetylation and increased stabilization of MTs in macrophages treated with LPS. Such acetylation positively amplified p38 MAP kinase signaling and stimulated production of IL-10 [34]. Meanwhile, ECTV-infected cells that were treated with LPS or left untreated showed extensive acetylation of MTs (Fig. 3A). To investigate whether increased acetylation correlates with increased MT stability, we examined the effect of nocodazole, a synthetic MT depolymerizing agent, on MT stability. After treatment with nocodazole for 5 or $30 \mathrm{~min}$, uninfected immature and mature GM-BM cells exhibited the complete disorganization of MT network (Fig. 3B). Meanwhile, ECTV-infected LPS-treated and untreated cells were more resistant to depolymerization by nocodazole and still showed the presence of MTs, especially within cellular extensions (Fig. 3B). Taken together, our data indicate that ECTV is able to stabilize MTs within immune cells, probably to facilitate the spread of the virus. Interestingly, our previous study showed that LPS treatment of ECTV-infected GM-BM cells derived from $\mathrm{BALB} / \mathrm{c}$ and $\mathrm{C} 57 \mathrm{BL} / 6$ mice increased both the percentage of $\mathrm{ECTV}^{+}$cells and the number of infectious virus particles at $24 \mathrm{hpi}$ when compared to LPS-untreated ECTV-infected cells [15]. Therefore, it is highly likely that LPS-induced extensive acetylation of MTs in ECTV-infected cells may additionally increase the dissemination of the virus. VACV also stabilizes MTs during later stages of infection in HeLa cells. It encodes two proteins with MAP-like activities, i.e., A10 and L4, which are engaged in generation of stable MTs [29]. Moreover, the F11 protein of VACV has been reported to stimulate MT growth by inhibition of RhoA signaling via the GTPase RhoA and its effector, $\mathrm{mDi}$, thus enhancing stabilization of MTs at the cell periphery. Therefore, it is suggested that changes in MT dynamics may enhance the spread of VACV by facilitating transport of viral particles to the plasma membrane for subsequent fusion and release [35].
In summary, ECTV is able to induce changes in the spatial organization and dynamics of MTs during its replication in immune cells, such as cDCs and macrophages. ECTV probably encodes proteins that function to generate stable MTs that serve different purposes during viral replication. This indicates adaptation of ECTV to its natural host immune cells and evolution of unique strategies by the virus to replicate and spread effectively even in highly specialized immune cells. Because MTs play a central role during viral replication and intracellular transport and regulate important processes critical to the outcome of infection, it is important to extend our knowledge of MT-virus interactions to exploit these elements as potential targets of antiviral therapy.

Acknowledgements We thank Roche Diagnostics Poland for help and assistance in real-time PCR experiments. Results were partially presented at the 7th Congress of European Microbiologists-FEMS 2017, 9-13 July 2017, Valencia, Spain, in which LS-D's participation was supported by the Leading National Research Centre (KNOW) Scientific Consortium "Healthy Animal - Safe Food," decision of Ministry of Science and Higher Education No. 05-1/KNOW2/2015.

Funding This work was supported by grants UMO-2012/05/D/ NZ6/02916 and UMO-2011/03/B/NZ6/03856 from the National Science Center in Cracow, Poland.

\section{Compliance with ethical standards}

Conflict of interest No conflict of interest is declared.

Ethical statement Experimental procedures on animals were approved by the 3rd Ethical Committee for Animal Experimentation at Warsaw University of Life Sciences - SGGW (permission no. 34/2012) and were performed in accordance with institutional guidelines for care and use of laboratory animals.

Open Access This article is distributed under the terms of the Creative Commons Attribution-NonCommercial 4.0 International License (http://creativecommons.org/licenses/by-nc/4.0/), which permits unrestricted use, distribution, and reproduction in any medium, provided you give appropriate credit to the original author(s) and the source, provide a link to the Creative Commons license, and indicate if changes were made.

\section{References}

1. Buller RM (2004) Mousepox: a small animal model for biodefense research. Appl Biosaf 9:10-19

2. Esteban D, Parker S, Schriewer J et al (2012) Mousepox, a small animal model of smallpox. Methods Mol Biol 890:177-198

3. Abrahao J, Oliveira TML, Campos RK et al (2009) Bovine vaccinia outbreaks: detection and isolation of vaccinia virus in milk samples. Foodborne Pathog Dis 6:1141-1146

4. Shchelkunov SN (2013) An increasing danger of zoonotic orthopoxvirus infections. PLoS Pathog 9:e1003756 
5. Laguardia-Nascimento M, de Oliveira APF, Azevedo IC et al (2017) Spread of poxviruses in livestock in Brazil associated with cases of double and triple infection. Arch Virol 162:2797-2801

6. van der Vaart B, Akhmanova A, Straube A (2009) Regulation of microtubule dynamic instability. Biochem Soc Trans 37:1007-1013

7. Naghavi MH, Walsh D (2017) Microtubule regulation and function during virus infection. J Virol 91:e00538-17

8. Ward BM, Moss B (2001) Vaccinia virus intracellular movement is associated with microtubules and independent of actin tails. $\mathrm{J}$ Virol 75:11651-11663

9. Szulc-Dabrowska L, Gregorczyk KP, Struzik J et al (2016) Remodeling of the fibroblast cytoskeletal architecture during the replication cycle of Ectromelia virus: a morphological in vitro study in a murine cell line. Cytoskeleton 7:396-417

10. Newsome TP, Marzook NB (2015) Viruses that ride on the coattails of actin nucleation. Semin Cell Dev Biol. 46:155-163

11. Drillien R, Spehner D, Bohbot A et al (2000) Vaccinia virusrelated events and phenotypic changes after infection of dendritic cells derived from human monocytes. Virology 268:471-481

12. Chahroudi A, Garber DA, Reeves P et al (2006) Differences and similarities in viral life cycle progression and host cell physiology after infection of human dendritic cells with modified vaccinia virus Ankara and vaccinia virus. J Virol 80:8469-8481

13. Hansen SJ, Rushton J, Dekonenko A et al (2011) Cowpox virus inhibits human dendritic cell immune function by nonlethal, nonproductive infection. Virology 412:411-425

14. Szulc-Dąbrowska L, Struzik J, Ostrowska A et al (2017) Functional paralysis of GM-CSF-derived bone marrow cells productively infected with ectromelia virus. PLoS One 12:0179166

15. Szulc-Dąbrowska L, Struzik J, Cymerys J et al (2017) The in vitro inhibitory effect of ectromelia virus infection on innate and adaptive immune properties of GM-CSF-derived bone marrow cells is mouse strain-independent. Front Microbiol 8:2539

16. Drabek K, van Ham M, Stepanova T et al (2006) Role of CLASP2 in microtubule stabilization and the regulation of persistent motility. Curr Biol 16:2259-2264

17. Galjart N (2005) CLIPs and CLASPs and cellular dynamics. Nat Rev Mol Cell Biol 6:487-498

18. Vaughan KT (2005) TIP maker and TIP marker; EB1 as a master controller of microtubule plus ends. J Cell Biol 171:197-200

19. Imaoka H, Toiyama $Y$, Saigusa $S$ et al (2015) RacGAP1 expression, increasing tumor malignant potential, as a predictive biomarker for lymph node metastasis and poor prognosis in colorectal cancer. Carcinogenesis 36:346-354

20. Rubin CI, Atweh GF (2004) The role of stathmin in the regulation of the cell cycle. J Cell Biochem 93:242-250

21. Mistry SJ, Bank A, Atweh GF (2007) Synergistic antiangiogenic effects of stathmin inhibition and taxol. Mol Cancer Res $5: 773-782$
22. Rauhala HE, Teppo S, Niemelä $S$ et al (2013) Silencing of the ARP2/3 complex disturbs pancreatic cancer cell migration. Anticancer Res 33:45-52

23. Wu C, Asokan SB, Berginski ME et al (2012) Arp2/3 is critical for lamellipodia and response to extracellular matrix cues but is dispensable for chemotaxis. Cell 148:973-987

24. Krause M, Dent EW, Bear JE et al (2003) Ena/VASP proteins: regulators of the actin cytoskeleton and cell migration. Annu Rev Cell Dev Biol 19:541-564

25. Bamburg JR, Bernstein BW (2010) Roles of ADF/cofilin in actin polymerization and beyond. F1000 Biol Rep 2:62

26. Chen QY, Xu W, Jiao DM et al (2013) Silence of ezrin modifies migration and actin cytoskeleton rearrangements and enhances chemosensitivity of lung cancer cells in vitro. Mol Cell Biochem 377:207-218

27. Szulc-Dąbrowska L, Gregorczyk KP, Struzik J, et al (2018) Long actin-based cellular protrusions as novel evidence of cytopathic effect induced in immune cells infected by ectromelia virus. Cent Eur J Immunol (Ahead of print)

28. Hollinshead M, Rodger G, Van Eijl H et al (2001) Vaccinia virus utilizes microtubules for movement to the cell surface. J Cell Biol 154:389-402

29. Ploubidou A, Moreau V, Ashman K et al (2000) Vaccinia virus infection disrupts microtubule organization and centrosome function. Embo J 19:3932-3944

30. Chen N, Danila MI, Feng Z et al (2003) The genomic sequence of ectromelia virus, the causative agent of mousepox. Virology 317:165-186

31. Lynn H, Horsington J, Ter LK et al (2012) Loss of cytoskeletal transport during egress critically attenuates Ectromelia virus infection in vivo. J Virol 86:7427-7443

32. Gregorczyk KP, Wyżewski Z, Szczepanowska J et al (2018) Ectromelia virus affects mitochondrial network morphology, distribution, and physiology in murine fibroblasts and macrophage cell line. Viruses 10:266

33. Naghavi MH, Gundersen GG, Walsh D (2013) Plus-end tracking proteins, CLASPs, and a viral Akt mimic regulate herpesvirusinduced stable microtubule formation and virus spread. Proc Natl Acad Sci USA 110:18268-18273

34. Wang B, Rao YH, Inoue $M$ et al (2014) Microtubule acetylation amplifies p38 kinase signaling and anti-inflammatory IL-10 production. Nat Commun 5:3479

35. Arakawa Y, Cordeiro JV, Way M (2007) F11L-mediated inhibition of RhoA-mDia signaling stimulates microtubule dynamics during vaccinia virus infection. Cell Host Microbe 1:213-226 